Manuscript number: COFS-D-15-00031

\title{
Genomics tools in microbial food safety
}

Matthew J. Stasiewicz ${ }^{\mathrm{a}}$, Henk C. den Bakker ${ }^{\mathrm{b}}$, and Martin Wiedmann ${ }^{\mathrm{c}}$

Note: 'den Bakker' is a family name

${ }^{a}$ Department of Food Science and Human Nutrition, College of Agriculture, Consumer and

Environmental Sciences. University of Illinois at Urbana-Champaign, Urbana, II 61801. USA

${ }^{\mathrm{b}}$ Department of Animal and Food Sciences, College of Agricultural Sciences and Natural Resources.

Texas Tech University, Lubbock, TX 79409. USA.

${ }^{\mathrm{c}}$ Department of Food Science, College of Agriculture and Life Sciences. Cornell University, Ithaca, NY 14853. USA.

E-mails: Stasiewicz, mstasiewicz@gmail.com.den Bakker, Henk.C.den-bakker@ttu.edu. Wiedmann, mw16@cornell.edu.

*Corresponding author:

Dr. Matthew Stasiewicz

Department of Food Science and Human Nutrition, College of Agriculture, Consumer and Environmental Sciences. University of Illinois at Urbana-Champaign, Urbana, II 61801. USA Phone: 313-212-7486

E-mail: mstasiewicz@gmail.com 
ABSTRACT

Genomics tools, and specifically whole-genome sequencing (WGS) methods, are rapidly transforming food safety, specifically through improved outbreak detection. In the U.S., whole genome sequencing of all human clinical Listeria monocytogenes isolates has been performed since 2013; isolates from foods and food processing plants obtained by regulatory agencies are also routinely characterized by WGS. Routine application of WGS appears to increase the number of listeriosis outbreaks that are detected and traced back to specific food sources. While rapidly improving WGS technologies have driven use of omics tools in food safety over the last 5 to 7 years, improved analytic methods will drive effective application of omics tools including genomics, transcriptomics, proteomics, and metabolomics over the next few years. 


\section{Introduction to omics approaches in microbial food safety.}

For food safety, the term "omics" encompasses a cornucopia of different methodologies including genomics, transcriptomics, proteomics, and metabolomics that, through advanced bioinformatics, provide insights relevant to reducing microbial food safety hazards [1]. Previous reviews have summarized application of these omics tools in food related applications using the term "foodomics" $[2,3]$. While many omics approaches are having a major impact on the methods of food safety research, genomics and whole genome sequencing (WGS) have been the most widely applied [1]. Specifically, WGS of bacterial isolates has started to replace other molecular methods, such as Pulse Field Gel Electrophoresis (PFGE), as subtyping method of choice. It appears that we are at the cusp of rapid and widespread application of WGS-based tools by regulatory and public health agencies as well as researchers. There is, however, an urgent need to facilitate use of omics tools, as well as the information generated by these tools, by the food industry and food safety professionals. This review is aimed to provide an overview of recent trends in the use of omics tools in microbial food safety (Figure 1), with an emphasis on genomics applications to bacterial foodborne pathogens.

\section{Evolution and current status of whole-genome sequencing tools.}

Whole-genome sequencing technology has progressed far beyond Sanger sequencing that produced the first compete bacterial genome sequence, of Haemophilus influenza, reported in 1995 [4], and the first human genome [5], reported in 2004 which cost almost 3 billion dollars and 13 years of work. Now a variety of next-generation sequencing tools exist that use alternatives to chain-terminating (Sanger) sequencing chemistry for high-throughput nucleotide sequence determination (reviewed in [6]), mainly (i) amplified template sequencing, which generate relatively shorter read lengths and (ii) single-molecule sequencing, which generate relatively longer read lengths. Each generates dramatically larger volumes 
of raw sequence data at a reduced per base cost. Choice of a particular sequencing platform for a project depends on the specific advantages and disadvantages of each platform.

Amplified template platforms, e.g. Illumina sequencers, are well-suited for experiments involving many strains and resequencing applications (when a closely related reference genome can guide analysis). These platforms generate millions of small (in the 100s of base-pairs) sequence reads. They have proven popular for improved molecular subtyping of foodborne pathogens [1]. These platforms provide economies of scale for centralized sequencing of small (<10 Mb), bacterial genomes. In retrospective sequencing of nearly 200 L. monocytogenes isolates from U.S. retail deli operations (M Stasiewicz, PhD thesis, Cornell University, 2015), high-output sequencing with multiplexing reagents for library preparation allows many (up to $96+$ ) isolates to be sequenced in parallel, generating WGS for $<\$ 100$ per isolate. Costs will likely drop further; custom protocols reduce costs of multiplexed library preparation from $>\$ 50$ to $<\$ 10$ per sample [7].

Single molecule platforms, particularly Single-Molecule Real-Time (SMRT) and nanopore-based sequencing platforms, e.g. PacBio and MinION sequencers respectively, are well-suited for comparative genomics questions that rely on finished genomes [8]. Using sequencing reads in the 10 of thousands of base pairs, these platforms allow for production of finished, closed, bacterial genomes through hybrid analysis along with short-read data [9]. Finished genomes can also be constructed from single-molecule data alone [10,11], including for a Salmonella Typhimurium isolated from chicken breast [12]. Illustrating value beyond genome assembly, a USB-operated nanopore sequencer has been shown to resolve genomic artifacts from short-read data, such as the structure and insertion site of an antimicrobial resistance island in Salmonella Typhi [13].

\section{Evolution and current status of bioinformatics tools used to analyze bacterial WGS data.}


Bioinformatics tools for bacterial WGS data perform the same basic tasks as were necessary before highthroughput WGS, e.g. genome assembly, comparison, and subtyping. The challenge is to adapt the tools to (i) primary data that are large collections of much shorter genome sequence fragments, and (ii) comparisons between increasingly larger numbers of whole genomes where manual, genome-bygenome, comparisons are impractical. These adaptations pose both computational and user-interface challenges (see introductory tutorials $[14,15])$.

Currently, two main strategies exist for the analysis of bacterial WGS data, including (i) whole genome Single Nucleotide Polymorphism (SNP) analysis, and (ii) whole genome, gene-based analyses such as whole-genome Multi Locus Sequence Typing (wgMLST). Typical workflows for each involve (i) sequencing quality control, (ii) possible genome assembly, (iii) variant calling and (iv) subtyping based on variants. Quality control tools can be automated by in-house pipelines [16], allowing the biologist to focus on downstream analysis. Whole genome mapping [17] and de novo assembly [18] tools are increasing in flexibility and speed. But phylogenetic and subtyping results can be estimated without the need for these time consuming steps, using tools including: k-mer based core- and pan-genome SNP detection (e.g., kSNP [19]); de Bruijn graph approaches to SNP, indel, and larger variant detection (e.g., Cortex [20]); and read mapping to only targets of interest for in silico MLST and gene detection (e.g., SRST2 [21]) and niche applications such as Salmonella serotype determination [22]. Whole-genome MLST, implemented in the Bacterial Isolate Genome Sequence Database (BIGSdb) [23] and applied to Campylobacter [24], provides an intermediate between highly discriminatory, less standardized wholegenome SNP-based phylogenetics and highly standardized, less discriminatory MLST. Other comparative genomics functions that have been scaled to the analysis of multiple microbial genomes include: annotation $[25,26]$, mobile element detection $[27,28]$ and recombination detection [29-31].

To facilitate adoption of modern bioinformatics tools, graphical, often web-based, user interfaces are allowing biologists without extensive programming skills to apply modern algorithms to their data. 
Examples include: the web-based generalist software Galaxy (http://galaxyproject.org/, [15,32]) specific bacterial WGS analyses hosted by the Center for Genomic Epidemiology (http://www.genomicepidemiology.org/), tools from sequencer companies (e.g. Basespace from Illumina, http://basespace.illumina.com/) and programs from software companies (e.g. Geneious http://www.geneious.com and Bionumerics http://www.applied-maths.com/bionumerics). While user interfaces are appropriate for analysis of single or small numbers of genomes, they are not yet well suited to high-throughput analyses due to challenges of moving large amounts of data between servers and the lack of analysis flexibility. Still, it is likely that cloud-based data storage and computationally less expensive analysis pipelines for food safety will be developed in the near future, as has been envisioned for clinical molecular diagnostics [33].

\section{Outbreak detection and surveillance using WGS.}

Retrospective and pilot studies on use of WGS to characterize bacterial foodborne pathogens associated with outbreaks or surveillance efforts have been published since approximately 2011 [34]. WGS-based subtyping provides for improved subtype discrimination over PFGE and other subtyping methods, which is particularly important for clonal pathogens. Work is ongoing to adapt WGS analyses to real-time outbreak detection and surveillance.

Two studies published in 2014 [35,36] specifically explored the use of WGS to provide improved subtype discrimination for Salmonella enterica serotype Enteritidis. This serotype represents a particularly important target for WGS-based subtyping as approx. $50 \%$ of human serotype Enteritidis isolates in the U.S. represent a single Xbal PFGE type with $85 \%$ of isolates classified into 5 PFGE types [36]. The study by den Bakker et al. [36] specifically showed that WGS will allow for improved detection of serotype Enteritidis outbreaks. Future, broad based implementation of WGS-based subtyping for Salmonella and 
specifically Salmonella Enteritidis, is thus likely to substantially increase the number of Salmonella outbreaks that are detected and recognized.

WGS also addresses the issue of "overdiscrimination" by PFGE; isolates that share a very recent common ancestor can show different PFGE patterns, which typically are caused by gain or loss of mobile elements such as prophage or plasmids (M Stasiewicz, PhD thesis, Cornell University, 2015). For example, isolates with two distinct PFGE patterns were linked to a listeriosis outbreak that occurred in Canada in 2008; WGS showed that isolates with these two PFGE types were closely related, likely shared a recent common ancestor, and a prophage accounted for the difference in PFGE type [37]. Similarly, the difference of PFGE type between two L. monocytogenes isolates linked to a listeriosis outbreak from cantaloupe was likely related to the gain or loss of a comK prophage [38]. Finally, variation in the presence or absence of distinct plasmids was related to variation in PFGE types among isolates from two listeriosis outbreaks linked to turkey deli meat, one in 1998-1999 and one in 2002 [39].

Routine use of WGS for surveillance has been implemented in 2013; in September of that year the U.S. CDC announced that all human L. monocytogenes isolates collected in the U.S. would be characterized by WGS [40]. Since implementation of routine WGS of human L. monocytogenes isolates, a number of small and medium size listeriosis outbreaks have been detected, ostensibly facilitated by use of WGS [41-45]. Two important features emerge from these outbreaks. First, retrospective WGS of isolates from 5 listeriosis cases from up to 5 years prior to a 2015 outbreak in ice cream was able to confirm those cases were related to the ongoing outbreak [42]. Second, both an outbreak linked to soft cheese [45] and one linked to bean sprouts [44] were initially identified not by classical epidemiological approaches but by comparing the WGS of L. monocytogenes from food samples collected during routine FDA inspection, and subsequent re-inspection, to routinely collected WGS of clinical L. monocytogenes isolates. 
Epidemiologic surveillance of foodborne pathogens using WGS will continue to expand. While the U.S. FDA GenomeTrakr project (http://www.fda.gov/Food/FoodScienceResearch/WholeGenomeSequencingProgramWGS/) began sequencing L. monocytogenes and Salmonella isolates obtained by regulatory agencies from foods and food processing plants, current efforts are expanding the network to cover (i) additional foodborne pathogens, E. coli, Campylobacter, Vibrio, Cronobacter, etc., and (ii) international scope, with labs in Argentina, Ireland, the U.K., Turkey, Belgium, and Mexico (as of 5/10/15, http://www.ncbi.nlm.nih.gov/bioproject query 'genometrakr').

\section{WGS beyond bacterial foodborne pathogens}

While the majority of applications of WGS in food safety have focused on bacterial pathogens, some work has been reported on application of WGS beyond bacterial foodborne pathogens. For example, WGS of Cryptosporidium parvum, C. hominis and C. muris have been reported [46-48]. Routine WGS of foodborne parasites, however, is not yet used for surveillance or outbreak investigations [1]. That previous review, published in 2014 [1], also summarized applications of WGS for detection and characterization of viral pathogens; in this case WGS is often used in metagenomics applications, including sequencing of total viral nucleic acids. In one outbreak, WGS of norovirus cDNA not only allowed successful delineation of the scope of a nosocomial outbreak, but phylogenetic analyses also allowed for establishment of the likely direction of transmission between two patients [49]. Continued development of whole-genome deep-sequencing methods for noroviruses and other viruses will provide approaches that allow for routine WGS-based virus surveillance [50], similar to WGS-based surveillance systems for bacterial pathogens.

\section{Other omics approaches in food safety}


Omics approaches other than genomics and WGS also are important tools and technology platforms in food safety, but are more typically used in research applications and have not yet moved to routine application to the same extent as WGS. As recently reviewed [1], metagenomics-based characterization of microbial communities provides a very promising and powerful approach to food safety testing, which allows for culture-independent identification, from food and environmental samples and clinical specimens, of non-culturable and difficult to detect foodborne disease causing agents [51]. Of note, the term metagenomics is often used to refer to two distinct approaches, including (i) 16S rRNA gene amplicon microbiome sequencing as well as (ii) "true" metagenomic sequencing where all DNA in a sample is sequenced. Potential concerns associated with the use of metagenomic approaches to detection of foodborne pathogens include the possibility of positive signals for pathogens due to presence of DNA in the absence of life organisms. In addition, 16S rRNA sequencing may not have the discriminatory power to reliably differentiate closely related pathogens and non-pathogens (e.g., L. monocytogenes and L. innocua [52]) and "true" metagenomic sequence assembly pipelines and analysis tools may also lead to misleading identification of organisms and pathogens that are not truly represent in a sample (see http://nickloman.github.io/2015/02/11/metagenomics-best-hit-analysis-caveatemptor/). While metagenomics tools thus show considerable potential for food safety applications, further development efforts are needed to fully realize the potential of these methods.

Transcriptomics, proteomics, and metabolomics approaches also have potential for food safety applications [1,2], but food safety applications are largely limited to research on foodborne pathogen biology, including research on development and discovery of new control strategies. For further details on metabolomics and transcriptomics tools and their application to food safety, see reviews by CastroPuyana and Herrero [53] and Valdés et al. [54], respectively. These approaches have the potential though to allow for characterization of the physiological state of pathogens in foods, which may lead to paradigm shifting approaches to food safety, where assessment of the physiological state of a pathogen 
in a food is considered when assessing to human health hazard posed by an organism present in a given food.

\section{Conclusion}

Whole genome sequencing has already transformed foodborne disease outbreak detection. Improved subtyping methods are able to detect increasingly smaller outbreaks and collaborations between clinical $(C D C)$ and regulatory (FDA) agencies have allowed for outbreaks to be identified through retrospective analysis of isolates collected during regulatory actions. Further improvements in genome sequencing technology and bioinformatics tools will allow food safety researchers to concentrate on asking critical biological questions rather than grappling with complex data sets. Omics approaches in food safety will continue to move beyond descriptive questions, such as how closely related one pathogen to another, to functional questions, such as how pathogens interact with their microbial and food matrix environment and how that knowledge can drive improvements in food safety.

\section{Acknowledgements}

Teaching activities related to food safety in the Wiedmann lab are supported by supported by the USDA National Institute of Food and Agriculture (NIFA) National Education Program in Food Safety 201167005-30010.

Any opinions, findings, conclusions, or recommendations expressed in this publication are those of the authors and do not necessarily reflect the view of (NIFA) or the United States Department of Agriculture (USDA).

\section{Figure Legend}

Figure 1. Flow diagram of current omics approaches in food safety, with an emphasis on whole genome sequencing of bacterial foodborne pathogens for improved surveillance. Each box groups a major class 
of inputs or outputs, with specific elements of those classes listed in smaller text. Information flows between elements are indicated by directional arrows. 


\section{Annotated References}

1. Bergholz TM, Moreno Switt AI, Wiedmann M: Omics approaches in food safety: fulfilling the promise? Trends Microbiol 2014, 22(5):275-281.

2. Giacometti J, Josic D: Foodomics in microbial safety. TrAC Trend Anal Chem 2013, 52(0):16-22.

3. Garcia-Canas V, Simo C, Herrero M, Ibanez E, Cifuentes A: Present and future challenges in food analysis: foodomics. Anal Chem 2012, 84(23):10150-10159.

4. Fleischmann R, Adams M, White O, Clayton R, Kirkness E, Kerlavage A, Bult C, Tomb J, Dougherty $B$, Merrick J, al. e: Whole-genome random sequencing and assembly of Haemophilus influenzae Rd. Science 1995, 269(5223):496-512.

5. Human Genome Sequencing C: Finishing the euchromatic sequence of the human genome. Nature 2004, 431(7011):931-945.

6. Loman NJ, Constantinidou C, Chan JZ, Halachev M, Sergeant M, Penn CW, Robinson ER, Pallen MJ: High-throughput bacterial genome sequencing: an embarrassment of choice, a world of opportunity. Nat Rev Microbiol 2012, 10(9):599-606.

7. Baym M, Kryazhimskiy S, Lieberman TD, Chung H, Desai MM, Kishony R: Inexpensive multiplexed library preparation for megabase-sized genomes. arXivorg 2015, http://dx.doi.org/10.1101/013771.

8. Schadt EE, Turner S, Kasarskis A: A window into third-generation sequencing. Human Molecular Genetics 2010, 19(R2):R227-R240.

9. Bashir A, Klammer AA, Robins WP, Chin C-S, Webster D, Paxinos E, Hsu D, Ashby M, Wang S, Peluso $P$, Sebra R et al: A hybrid approach for the automated finishing of bacterial genomes. Nat Biotech 2012, 30(7):701-707.

10. Chin C-S, Alexander DH, Marks P, Klammer AA, Drake J, Heiner C, Clum A, Copeland A, Huddleston J, Eichler EE, Turner SW et al: Nonhybrid, finished microbial genome assemblies from long-read SMRT sequencing data. Nat Meth 2013, 10(6):563-569.

11. Koren S, Harhay G, Smith T, Bono J, Harhay D, Mcvey S, Radune D, Bergman N, Phillippy A: Reducing assembly complexity of microbial genomes with single-molecule sequencing. Genome Biol 2013, 14(9):R101.

12. Hoffmann M, Muruvanda T, Allard MW, Korlach J, Roberts RJ, Timme R, Payne J, McDermott PF, Evans P, Meng J, Brown EW et al: Complete genome sequence of a multidrug-resistant 
Salmonella enterica serovar Typhimurium var. 5- strain isolated from chicken breast. Genome Announc 2013, 1(6).

13. ${ }^{* *}$ Ashton PM, Nair S, Dallman T, Rubino S, Rabsch W, Mwaigwisya S, Wain J, O'Grady J: MinION nanopore sequencing identifies the position and structure of a bacterial antibiotic resistance island. Nat Biotechnol 2015, 33(3):296-300.

The authors identify the structure and chromosomal insertion site of a antimicrobial resistance island in Salmonella Typhi haplotype H58 using a hybrid assembly of long-read nanopore data and short-read Illumina data. The long-read sequencing platform (Minion) used in this study has a much lower capital cost and smaller footprint than existing long-read sequencers (from PacBio), suggesting that long-read sequencing may soon be affordable for non-specialist laboratories.

14. Edwards DJ, Holt KE: Beginner's guide to comparative bacterial genome analysis using nextgeneration sequence data. Microb Inform Exp 2013, 3(1):2.

15. Blankenberg D, Taylor J, Nekrutenko A: Online resources for genomic analysis using highthroughput sequencing. Cold Spring Harb Protoc 2015, 2015(4):doi:10.1101/pdb.top083667.

16. Paszkiewicz KH, Farbos A, O'Neill P, Moore K: Quality control on the frontier. Front Genet 2014, $\mathbf{5}(157$.

17. Li H: Aligning sequence reads, clone sequences and assembly contigs with BWA-MEM. arXivorg 2013, http://arxiv.org/abs/1303.3997v1302.

18. Bankevich A, Nurk S, Antipov D, Gurevich AA, Dvorkin M, Kulikov AS, Lesin VM, Nikolenko SI, Pham S, Prjibelski AD, Pyshkin AV et al: SPAdes: a new genome assembly algorithm and its applications to single-cell sequencing. J Comput Biol 2012, 19(5):455-477.

19. Gardner SN, Hall BG: When whole-genome alignments just won't work: kSNP v2 software for alignment-free SNP discovery and phylogenetics of hundreds of microbial genomes. PLOS ONE 2013, 8(12):e81760.

20. Iqbal Z, Turner I, McVean G: High-throughput microbial population genomics using the Cortex variation assembler. Bioinformatics 2012, 1-2.

21. Inouye M, Dashnow H, Raven L, Schultz MB, Pope BJ, Tomita T, Zobel J, Holt KE: SRST2: Rapid genomic surveillance for public health and hospital microbiology labs. Genome Med 2014, 6(90).

22. *Zhang S, Yin Y, Jones MB, Zhang Z, Deatherage Kaiser BL, Dinsmore BA, Fitzgerald C, Fields PI, Deng $X$ : Salmonella serotype determination utilizing high-throughput genome sequencing data. J Clin Microbiol 2015, 53(5):1685-1692. 
The authors developed a web-based bioinformatics tool that was $>90 \%$ accurate at determining Salmonella serotype from WGS data using direct targets of $\mathrm{O}$ and $\mathrm{H}$ antigens (the $\mathrm{rfb}$ gene cluster and fliB and flic gene alleles, respectively). This tool provides an in silico method for comparing new Salmonella isolates to historic Salmonella serotype information.

23. Maiden MC, van Rensburg MJ, Bray JE, Earle SG, Ford SA, Jolley KA, McCarthy ND: MLST revisited: the gene-by-gene approach to bacterial genomics. Nat Rev Microbiol 2013, 11(10):728-736.

24. *Meric G, Yahara K, Mageiros L, Pascoe B, Maiden MC, Jolley KA, Sheppard SK: A reference pangenome approach to comparative bacterial genomics: identification of novel epidemiological markers in pathogenic Campylobacter. PLOS ONE 2014, 9(3):e92798.

The authors use a combination of 7 reference Campylobacter genomes to define a set of pan-genome genes, and then use that set to find, among 192 isolates, 21 and 27 genes present only in C. coli and jejuni, respectively. This multi-reference pan-genome approach provides a computationally tractable method to carry out gene-by-gene presence/absence analysis on hundreds of genomes without limiting results to only those genes that happen to be present in the chosen, traditionally single, reference genome.

25. Seemann T: Prokka: rapid prokaryotic genome annotation. Bioinformatics 2014, 30(14):20692069 .

26. Tatusova T, DiCuccio M, Badretdin A, Chetvernin V, Ciufo S, Li W: Prokaryotic genome annotation pipeline. In: The NCBI Handbook [Internet]. 2nd edition. National Center for Biotechnology Information, 2013:http://www.ncbi.nlm.nih.gov/books/NBK174280/.

27. Akhter S, Aziz RK, Edwards RA: PhiSpy: a novel algorithm for finding prophages in bacterial genomes that combines similarity- and composition-based strategies. Nucleic Acids Res 2012, 40(16):e126.

28. Carattoli A, Zankari E, Garcia-Fernandez A, Voldby Larsen M, Lund O, Villa L, Moller Aarestrup F, Hasman $\mathrm{H}$ : In silico detection and typing of plasmids using PlasmidFinder and plasmid multilocus sequence typing. Antimicrob Agents Chemother 2014, 58(7):3895-3903.

29. Croucher NJ, Page AJ, Connor TR, Delaney AJ, Keane JA, Bentley SD, Parkhill J, Harris SR: Rapid phylogenetic analysis of large samples of recombinant bacterial whole genome sequences using Gubbins. Nucleic Acids Res 2015, 43(3):e15:13pgs.

30. Didelot $X$, Wilson DJ: ClonalFrameML: efficient inference of recombination in whole bacterial genomes. PLoS Comput Biol 2015, 11(2):e1004041. 
31. Marttinen P, Hanage WP, Croucher NJ, Connor TR, Harris SR, Bentley SD, Corander J: Detection of recombination events in bacterial genomes from large population samples. Nucleic Acids Res 2012, 40(1):e6.

32. Goecks J, Nekrutenko A, Taylor J: Galaxy: a comprehensive approach for supporting accessible, reproducible, and transparent computational research in the life sciences. Genome Biol 2010, 11(8):R86.

33. Fricke WF, Rasko DA: Bacterial genome sequencing in the clinic: bioinformatic challenges and solutions. Nat Rev Genet 2014, 15(1):49-55.

34. Lienau EK, Strain E, Wang C, Zheng J, Ottesen AR, Keys CE, Hammack TS, Musser SM, Brown EW, Allard MW, Cao G et al: Identification of a salmonellosis outbreak by means of molecular sequencing. N Engl J Med 2011, 364(10):981-982.

35. Deng X, Desai PT, Den Bakker HC, Mikoleit M, Tolar B, Trees E, Hendriksen RS, Frye JG, Porwollik S, Weimer BC, Wiedmann M et al: Genomic epidemiology of Salmonella enterica serotype enteritidis based on population structure of prevalent lineages. Emerg Infect Dis 2014, 20(9):1481-1489.

36. **den Bakker HC, Allard MW, Bopp D, Brown EW, Fontana J, Iqbal Z, Kinney A, Limberger R, Musser KA, Shudt M, Strain E et al: Rapid whole-genome sequencing for surveillance of Salmonella enterica serovar enteritidis. Emerg Infect Dis 2014, 20(4):1306-1314.

In a two-part study, analysis of WGS data from (i) 35 retrospectively sequenced Salmonella Enteritidis isolates showed that SNP-based phylogenetic cluster analysis improved upon existing molecular subtyping methods and (ii) 58 prospectively sequenced Salmonella Enteritidis showed well-supported phylogenetic clusters of isolates all with the same PFGE type. The combined sequencing and bioinformatics workflow for SNP detection and phylogenetics was able to complete analysis of 32 isolates in less than 8 days, demonstrating that WGS subtyping analyses can be completed in time frames relevant to outbreak investigations.

37. Gilmour MW, Graham M, Van Domselaar G, Tyler S, Kent H, Trout-Yakel KM, Larios O, Allen V, Lee $B$, Nadon C: High-throughput genome sequencing of two Listeria monocytogenes clinical isolates during a large foodborne outbreak. BMC Genomics 2010, 11(120.

38. Lomonaco S, Verghese B, Gerner-Smidt P, Tarr C, Gladney L, Joseph L, Katz L, Turnsek M, Frace $\mathrm{M}$, Chen $\mathrm{Y}$, Brown E et al: Novel epidemic clones of Listeria monocytogenes, United States, 2011. Emerg Infect Dis 2013, 19(1):147-150.

39. Ying C, Robin MS, Sophia K: Genomic divisions/lineages, epidemic clones, and population structure. In: Handbook of Listeria Monocytogenes. CRC Press, 2008:337-357. 
40. Sauders B. CDC begins real-time whole genome sequencing of Listeira monocytogenes: 2013. http://aphltech.org/2013/11/25/cdc-begins-real-time-whole-genome-sequencing-of-listeriamonocytogenes/. Accessed 5/10/15.

41. CDC. Multistate Outbreak of Listeriosis Linked to Roos Foods Dairy Products (Final Update): 2014. http://www.cdc.gov/listeria/outbreaks/cheese-02-14/index.html. Accessed 5/10/15.

42. Matacic C: Sequencing finds listeria in unlikely places. Science Insider 2015, DOI: 10.1126/science.aac4551.

43. CDC. Multistate Outbreak of Listeriosis Linked to Commercially Produced, Prepackaged Caramel Apples Made from Bidart Bros. Apples (Final Update): 2015.

http://www.cdc.gov/listeria/outbreaks/caramel-apples-12-14/index.html. Accessed 5/10/15.

44. CDC. Wholesome Soy Products, Inc. Sprouts and Investigation of Human Listeriosis Cases (Final Update): 2015. http://www.cdc.gov/listeria/outbreaks/bean-sprouts-11-14/index.html. Accessed 5/10/15.

45. CDC. Oasis Brands, Inc. Cheese Recalls and Investigation of Human Listeriosis Cases (Final Update): 2014. http://www.cdc.gov/listeria/outbreaks/cheese-10-14/index.html. Accessed $5 / 10 / 15$.

46. Abrahamsen MS, Templeton TJ, Enomoto S, Abrahante JE, Zhu G, Lancto CA, Deng M, Liu C, Widmer G, Tzipori S, Buck GA et al: Complete genome sequence of the apicomplexan, Cryptosporidium parvum. Science 2004, 304(5669):441-445.

47. Xu P, Widmer G, Wang Y, Ozaki LS, Alves JM, Serrano MG, Puiu D, Manque P, Akiyoshi D, Mackey AJ, Pearson WR et al: The genome of Cryptosporidium hominis. Nature 2004, 431(7012):11071112.

48. Widmer $G$, Lee $Y$, Hunt $P$, Martinelli A, Tolkoff $M$, Bodi $K$ : Comparative genome analysis of two Cryptosporidium parvum isolates with different host range. Infect Genet Evol 2012, 12(6):12131221.

49. Kundu S, Lockwood J, Depledge DP, Chaudhry Y, Aston A, Rao K, Hartley JC, Goodfellow I, Breuer $\mathrm{J}$ : Next-generation whole genome sequencing identifies the direction of norovirus transmission in linked patients. Clin Infect Dis 2013, 57(3):407-414.

50. Cotten M, Petrova V, Phan MV, Rabaa MA, Watson SJ, Ong SH, Kellam P, Baker S: Deep sequencing of norovirus genomes defines evolutionary patterns in an urban tropical setting. $J$ Virol 2014, 88(19):11056-11069. 
51. Miller RR, Montoya V, Gardy JL, Patrick DM, Tang P: Metagenomics for pathogen detection in public health. Genome Med 2013, 5(9):81.

52. Collins MD, Wallbanks S, Lane DJ, Shah J, Nietupski R, Smida J, Dorsch M, Stackebrandt E: Phylogenetic analysis of the genus Listeria based on reverse transcriptase sequencing of $16 \mathrm{~S}$ rRNA. Int J Syst Bacteriol 1991, 41(2):240-246.

53. Castro-Puyana M, Herrero M: Metabolomics approaches based on mass spectrometry for food safety, quality and traceability. TrAC Trend Anal Chem 2013, 52(0):74-87.

54. Valdés A, Ibáñez C, Simó C, García-Cañas V: Recent transcriptomics advances and emerging applications in food science. TrAC Trend Anal Chem 2013, 52(0):142-154. 
